\title{
Solución Metaheurística para el Problema de Enrutamiento (RWA) en Redes de Ópticas de Multiplexación por División de Longitud de Onda (WDM)
}

\author{
Arturo B. Rodriguez ${ }^{(1)}$, Leonardo J. Ramirez ${ }^{(2)}$ y Felipe R. M. Basile ${ }^{(3)}$ \\ (1) Universidad Santiago de Chile, Departamento de Tecnología Industrial, Grupo de Investigación en \\ Nuevas Tecnologías (GINT), Santiago-Chile. (email: arturo.rodriguez@usach.cl) \\ (2) Universidad Militar de Nueva Granada, Grupo de Investigación en Telemedicina (TIGUM), Bogotá, \\ Colombia. (email: leonardo.ramirez@unimilitar.edu.co) \\ (3) Instituto Federal de Educación, Ciencia y Tecnología de Sao Paulo, Campus Pirituba, Brasil. \\ (email: felipe.basile@ifsp.edu.br)
}

Recibido May. 15, 2017; Aceptado Jul. 19, 2017; Versión final Ago. 10, 2017, Publicado Dic. 2017

\begin{abstract}
Resumen
El artículo muestra los resultados de una nueva metaheurística basada en el algoritmo Snake One que se ha denominado Snake Three. Se basa en la utilización de los enlaces hasta la lograr su congestión máxima. De esta forma se logra cierto orden de uso de los recursos dejando libre el resto de enlaces para satisfacer la demanda entrante o futura. La red utilizada en la simulación es la NSFNET de los Estados Unidos bajo parametrización similar a otras simulaciones, logrando estresar el sistema hasta los 180 Erlangs. Los resultados muestran una mejora en el indicador utilización de la red, aunque el indicador probabilidad de bloqueo no muestra mejora. La metaheurística Snake Three es la última de la serie Snake y ha demostrado su eficacia para poder ser utilizada en otros escenarios de multiplexación.
\end{abstract}

Palabras clave: algoritmos heurísticos; algoritmos snake; redes ópticas; RWA; WDM

\section{Metaheuristic Solution for the Routing and Wavelength Assignment (RWA) Problem in Wavelength-Division Multiplexing (WDM) Optical Networks}

\begin{abstract}
The article shows the results of a new metaheuristic based on the Snake One algorithm that has been called Snake Three. It is based on the use of links until achieving maximum congestion. In this way, some order in the use of resources is achieved by leaving free the rest of the links to satisfy the incoming or future demand. The network used in the simulation is the NSFNET, of the United States, under parameterization similar to other simulations, achieving to stress the system up to 180 Erlangs. The results show improvement in the indicator utilization of the network, while the indicator probability of blocking does not show improvement. The Snake Three metaheuristic is the latest in the Snake series and has proved its effectiveness to be used in other multiplexing scenarios.
\end{abstract}

Keywords: heuristics algorithm; snake algorithms; optical networks; RWA; WDM 


\section{INTRODUCCIÓN}

Las velocidades de transporte en las redes ópticas se han incrementado gracias a la tecnología WDM (Wavelength Division Multiplexing) donde cada longitud de onda alcanza hasta 2.5 Gbps y multiplicada por esta tecnología alcanza tantas veces como longitudes de onda se inserten en la misma fibra óptica monomodo. Por otro lado, se alcanzan tasas de $10 \mathrm{Gbps}, 25 \mathrm{Gbps}$ y otras tasas basadas en transmisión coherente. Los problemas asociados a estas redes radican en la obtención de rutas y la selección de la longitud de onda asociada, si se utiliza una misma longitud de onda para cada enlace de la ruta se trata de redes sin conversión de longitud de onda, y si se utilizan longitudes de onda diferentes en cada longitud de onda enlace de la ruta se trata de redes con conversión de longitud de onda, es decir los dispositivos de conmutación OXC (Optical Cross Connect) deben tener la capacidad de conversión en cada caso.

Las redes actuales inclusive tienen capacidad para conmutar fibras, bandas de longitudes de onda (waveband) y longitudes de onda. Los algoritmos utilizados en estas redes son de tipo heurístico y metaheurístico a diferencia de los dispositivos de enrutamiento electrónico donde se desarrollaron una serie de algoritmos tales como: Dikstra, Bellman-Ford, Floyd Warshall, Ford-Fulkerson entre otros, que se pueden denominar convencionales, estos últimos buscan la optimización basada en el vector distancia o sistema de costos. Sin embargo, los algoritmos heurísticos no son capaces de optimizar si el universo de soluciones es de un alto dinamismo y en tráfico variable (Rodriguez et al., 2014; Rodriguez et al. 2015). Los escenarios de tráfico dinámico no pueden ser resueltos por algoritmos optimizadores debido a que el universo de solución varía constantemente. Sin embargo, la heurística permite encontrar soluciones que no son necesariamente óptimas pero si son buenas soluciones, y no solo una solución sino varias en el mismo proceso algorítmico, dándole robustez a estos algoritmos.

Se han estudiado heurísticas y meta heurísticas tales como: Algoritmos genéticos, Simulated Annealing, Tabu Search, Luciérnaga, entre otros. Además, se han desarrollado diferentes meta heurísticas que modifican o perfeccionan las heurísticas a través de combinaciones o inclusión de procesos dentro de los algoritmos originales mejorando el tiempo de obtención de la solución, mayor cantidad de soluciones o dirigir las soluciones hacia un espacio del universo preestablecido o deseable. En ámbito de las redes ópticas WDM (Wavelength Division Multiplexing) la tecnología predominante es el switching que permite conmutar diferentes canales entre longitudes de onda, bandas de longitudes de onda, fibras, canales de tiempo, etc. (Zheng et al., 2016; Sadiq etal., 2016; Gutierrez et al., 2016). Los algoritmos heurísticos responden con mejores resultados para tráfico dinámico en este tipo de redes, sin embargo es muy difícil lograr una mejora en la probabilidad de bloqueo y la utilización de la red de manera simultánea. En la mayoría de trabajos el tráfico es modelado con una distribución poisson y la carga se da en erlangs, siendo el tráfico estático o dinámico se logra establecer una relación entre el tipo de tráfico y la relación existente entre el tiempo promedio entre llegadas de solicitudes de servicio y el tiempo promedio de solicitudes de conexión.

La presente investigación se muestra varias heurísticas y desarrolla la comparación de simulaciones hasta los 180 Erlangs en la red NSFNET (National Science Foundation Network), mostrando una nueva meta heurísticas basada en el algoritmo Snake One y muestra los resultados para la meta heurística Snake Three (SNK3), comparada con las heurísticas algoritmo genético (AG), Simulated Annealing (SA), algoritmo de referencia, Tabu Search (TS),Snake One (SNK1), Snake Two (SNK2) (Zang et al., 2001;Rodriguez et al., 2015; Rodriguez et al., 2018) y otros aplicados a diferentes áreas (Alarcón-Aquino et al., 2005; Russo et al., 2011; Piedrahita et al., 2014).

\section{ENCAMINAMIENTO EN REDES ÓPTICAS}

En las redes ópticas se presenta el problema RWA (Routing Wavelength Assignment) el mismo se define como la problemática de encontrar una ruta y longitud de onda para cada enlace de la ruta. En las redes ópticas se puede distinguir las redes sin conversión de longitud de onda (lambda) donde cada enlace tiene la misma lambda y las redes con conversión de la longitud de onda donde cada enlace puede tener diferente lambda.

La condición de usar la misma lambda es conocida en la literatura como restricción de continuidad de longitud de Onda o CCW (Constraint Continuity Wavelength) y para el otro caso se denomina reutilización de longitud de onda o WR (Wavelength Reuse). Un camino de luz o Lightpath (LP) es el conjunto de enlaces que conforman la ruta y una longitud onda asociada para el caso CCW. Por ejemplo una ruta del nodo 2 al nodo 5 para un sistema de 8 nodos etiquetados del 0 al 7, con 3 longitudes de onda por enlace $\left(\lambda_{0}, \lambda_{1}, \lambda_{2}\right)$, y se puede observar en la figura 1. 


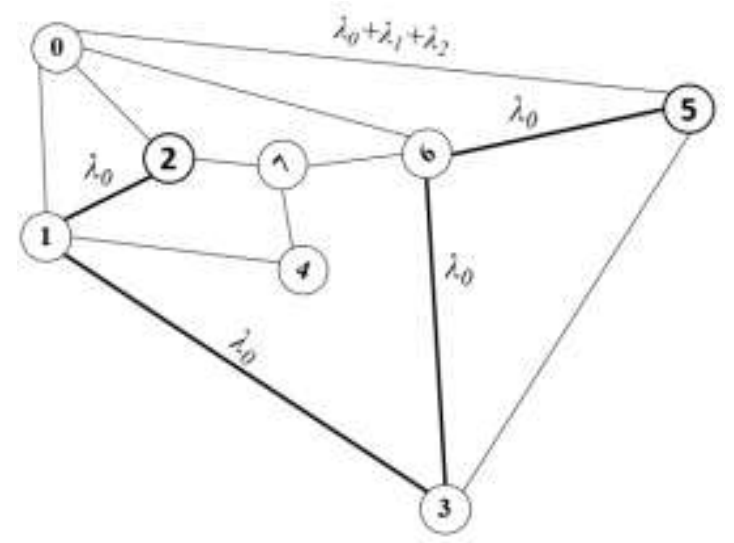

Fig. 1: Red óptica de ejemplo

$L P=\left(R, \lambda_{1}\right)=\left(e_{21}-e_{13}-e_{36}-e_{65}, \lambda_{0}\right)$

$L P=\left(e_{21}, \lambda_{1}\right)-\left(e_{13}, \lambda_{0}\right)-\left(e_{36}, \lambda_{1}\right)-\left(e_{65}, \lambda_{2}\right)$

Dónde:

$$
\begin{aligned}
& e_{i j}=\text { Enlace del nodo } i-\text { ésimo al nodo } j-\text { ésimo } \\
& R=e_{21}-e_{13}-e_{36}-e_{65}=\text { Ruta }
\end{aligned}
$$

La ecuación 1, muestra el lightpath para el caso CCW y la ecuación 2 para el caso WR, nótese en el último caso que para cada enlace existe una longitud de onda asociada distinta. Las redes WDM-CCW al utilizar la misma longitud de onda en cada enlace tienen una baja latencia en el establecimiento de la conexión, sin embargo el universo de soluciones se agota con rapidez cuando el tráfico aumenta generando una alta congestión en la red. Por otro lado, las redes WDM-WR tienen una utilización eficiente de los recursos de la red pero tienen una alta latencia en el establecimiento de la conexión. Se han utilizado diferentes estrategias en el campo heurístico y metaheruístico, y se pueden clasificar en dos, aquellas que dividen el problema en dos partes (ESP - Estrategia de Subdivisión del Problema) y aquellos que lo solucionan de manera integral (ESI - Estrategia de Solución Intergral). La ESP tiene la ventaja de disminuir el tiempo de obtención de la solución al simplificar el problema utilizando dos procesos algorítmicos, sin embargo al ser utilizado en redes WDM-CCW la asignación de longitud de onda se convierte en un obstáculo, por lo general mejoran el uso de la red pero aumentan la probabilidad de bloqueo. La ESI utiliza un solo proceso algorítmico para obtener el lightpath balanceando equilibradamente la utilización de la red y la probabilidad de bloqueo, sin embargo el tiempo de obtención de la solución es significativamente mayor que la ESP. Por otro lado se utilizan criterios de maximización o minimización de la función aptitud (fitness) por ejemplo, el retardo en el enlace, el ruido ASE de los amplificadores en la ruta, cantidad de canales utilizados en el enlace, congestión en el enlace, congestión en el enlace más utilizado, etc. basados en una métrica denominada costo, que se caracteriza por un número entero que representa los criterios antes mencionados.

\section{HEURÍSTICAS Y META HEURÍSTICAS}

Se define una topología de $\mathrm{N}$ conmutadores ópticos (OXC) etiquetados de 0 a $N-1$, con $n_{w}$ longitudes de onda por cada enlace de fibra óptica tal como $\left(\lambda_{0}, \lambda_{1}, \lambda_{2}, \lambda_{3}, \ldots \ldots, \lambda_{n_{W^{-1}}}\right)$ donde la solicitud de servicio entrante que llega a los nodos es (Ver ecuación 3):

$R_{i}^{j}=\left(D, n_{C}, t_{C}\right)$

\section{Dónde:}

$R_{i}^{j}$ es la $i$-ésima solicitud del $j$-ésimo nodo; $D$ es el nodo destino de la $i$-ésima solicitud del $j$-ésimo nodo; $n_{C}$ es el número de conexiones solicitadas; y $t_{C}$ es el tiempo de conexión solicitado - Holding Time.

En la Figura 2, se muestra la red anteriormente descrita, donde el nodo 2 es solicitado con $\mathrm{R}$ por una ruta hacia el nodo 5 con un canal por un tiempo de $500 \mathrm{~ms}$. Dicha red utiliza 3 longitudes de onda $\left(N=8, n_{W}=\right.$ 3) etiquetadas cada una con números del 0 al 7 . Por lo tanto se deberá buscar una ruta y una longitud de onda que permita satisfacer la demanda en condiciones de CCW, es decir la misma longitud de onda en cada enlace de la ruta. 


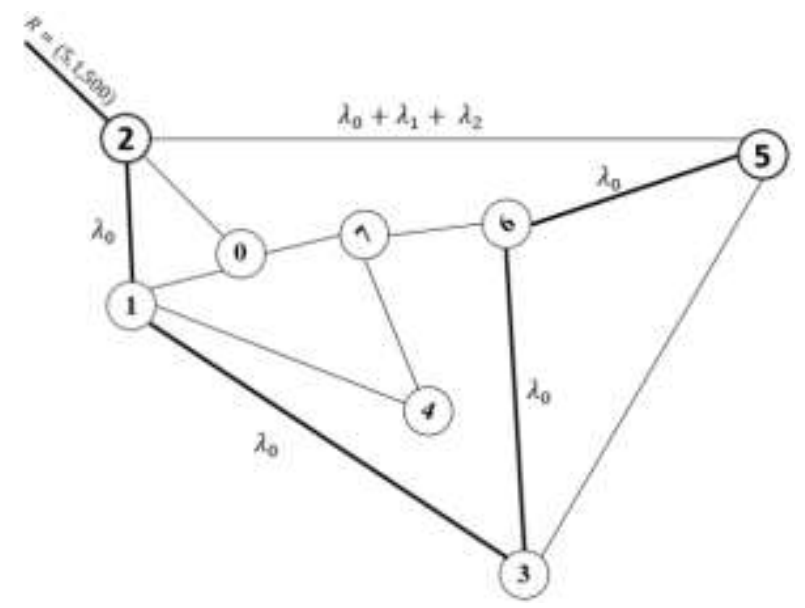

Fig. 2: Red óptica mostrando la solicitud de entrada.

Las heurísticas encuentran las soluciones a partir de un grupo aleatorio de elementos generado al inicio del algoritmo y después se realizan procedimientos propios de cada algoritmia, este grupo es denominado población, y está definida por:

$P_{0}=\left\{\begin{array}{c}p_{i j k} / p_{i 0 k}=\text { Origen } \wedge p_{i(N-1) k}=\text { Destino } \wedge p_{i j k}=\operatorname{Random}(0, N-1) \forall i \in[0, N-1] \\ j \in[1, N-2] \cap k \in\left[0, n_{W}-1\right]\end{array}\right\}$

Aquí, $P_{0}$ es la población inicial; y $p_{i j k}$ : es el elemento poblacional j-ésimo de la $i$-ésima solución para $k$ ésima longitud de onda.

Por ejemplo, en la matriz poblacional los elementos $p_{i 02}$ y $p_{i 72}$ corresponden al nodo origen y nodo destino del problema RWA donde $i \in[0,7]$. En la ecuación 5, se muestra la población inicial para la longitud de onda $\lambda_{2}(k=2)$, donde el nodo 2 recibe la solicitud de servicio hacia el nodo 5 .

$P_{0}=\left\{\begin{array}{llllllll}2 & 0 & 3 & 4 & 2 & 5 & 7 & 5 \\ 2 & 3 & 1 & 7 & 4 & 2 & 1 & 5 \\ 2 & 6 & 0 & 2 & 7 & 5 & 6 & 5 \\ 2 & 2 & 1 & 0 & 4 & 3 & 2 & 5 \\ 2 & 7 & 3 & 1 & 5 & 4 & 3 & 5 \\ 2 & 6 & 4 & 0 & 3 & 1 & 6 & 5 \\ 2 & 2 & 7 & 2 & 3 & 0 & 5 & 5 \\ 2 & 5 & 3 & 6 & 4 & 1 & 7 & 5\end{array}\right\}$

Para el caso de la población de la ecuación 5, se espera encontrar una ruta del nodo 2 al nodo 5 a través de la longitud de onda 2. Observando las rutas (filas) estas inicialmente no tienen un costo finito, por ejemplo la fila 0 o primera fila $2-0-3-4-2-5-7-5$ tiene el costo infinito debido a que tiene enlaces que no existen como $0-3$ y $7-5$. Sin embargo realizando procesos con la matriz poblacional según la heurística utilizada, estas filas pueden mejorar en aptitud y convirtiéndolas en finitas, lo que determina su candidatura como posible solución, cabe indicar que las nuevas filas solo serán buenas rutas y no necesariamente la óptima.

\section{Algoritmos Genéticos (AG)}

El AG es uno de los procesos más estudiados en la literatura su aplicación data de 1970 a través de John Henry Holland, se ha aplicado en diferentes áreas donde se requiere optimización, sin embargo desde la inserción de la fibra óptica en las redes de transporte, su utilización se ha intensificado a nivel investigativo. El algoritmo se basa en la combinación genética para obtener poblaciones con mejores características en cada evolución de la matriz poblacional, de esta forma se obtienen genotipos cada vez más aptos para la solución, y por lo general se realiza a través de una función denominada función de aptitud o fitness function. Además, se incorporan procesos de reproducción, mortandad y mutación similares a los producidos en la genética

De la ecuación 5 se puede extraer los cromosomas (filas) consecutivas con todos sus elementos (genes), tal como la ecuación 6: 
Ambos cromosomas se reproducirán al combinarse tal como se muestra en la ecuación 7:

$\left\{\begin{array}{llllllll}2 & 0 & 3 & 4 & 4 & 2 & 1 & 5 \\ 2 & 3 & 1 & 7 & 2 & 5 & 7 & 5\end{array}\right\}$

Esta nuevas filas tienen nuevas aptitudes, es decir una función de aptitud diferente a las filas que la generaron, produciéndose una nueva población, luego se procede a un ordenamiento descendente por la FF (Fitness Function) y las filas de mayor valor quedarán debajo de la población, y estás podrán ser eliminadas (mortandad) y repuestas con nuevas filas aleatorias (natalidad), también se puede cambiar un elemento (gen) en particular de manera aleatoria (mutación), para lograr minimizar la FF hasta que la aptitud sea la deseada. Trabajos como los mostrados en (Rodríguez et al., Cheng etal., 2016; Zahedi et al., 2016; Lefever et al., 2016) entregan resultados que garantizan un excelente funcionamiento bajo tráfico dinámico y con alta carga de stress.

\section{Simulated Annealing (SA)}

El algoritmo SA, es una heurística que desarrolla un proceso similar a la técnica de calentamiento y enfriamiento lento con la finalidad de cambiar las características físicas de los materiales. Existe discusión respecto de quien introdujo el método, los autores Scott Kirkpatrick, C. Daniel Gelatt y Mario P. Vecchi en el 1983 mencionan que existe una profunda conexión entre la mecánica estadística y la optimización combinatoria multivariable y Vlado Černý en 1985, menciona que por analogía con la termodinámica estadística y utilizando la probabilidad dada por la distribución de Boltzmann-Gibbs se puede llegar a estar muy cerca de la solución óptima de un problema hasta se puede obtener el óptimo verdadero (Kirkpatrick et al., 1983; Černý et al., 1985). Para este caso la población se trata como un conjunto de moléculas que rotan similarmente a los gases dentro de un volumen de control, donde las paredes son los elementos $p_{i 02}$ y $p_{i(N-1) 2}$, de tal forma que el resto de elementos rotan por capas a velocidades diferentes siendo más lenta afuera y rápida dentro y esta velocidad va disminuyendo según una función de enfriamiento tal como se ve en la ecuación 5:

$v_{i}=v_{i 0}(1-\propto T)$

En esta ecuación, $v_{i}$ es la velocidad de la capa $i$-ésima a temperatura $\mathrm{T} ; v_{i 0}$ es la velocidad inicial de la capa $i$-ésima; $\propto$ es el coeficiente de enfriamiento; y $T$ es la temperatura.

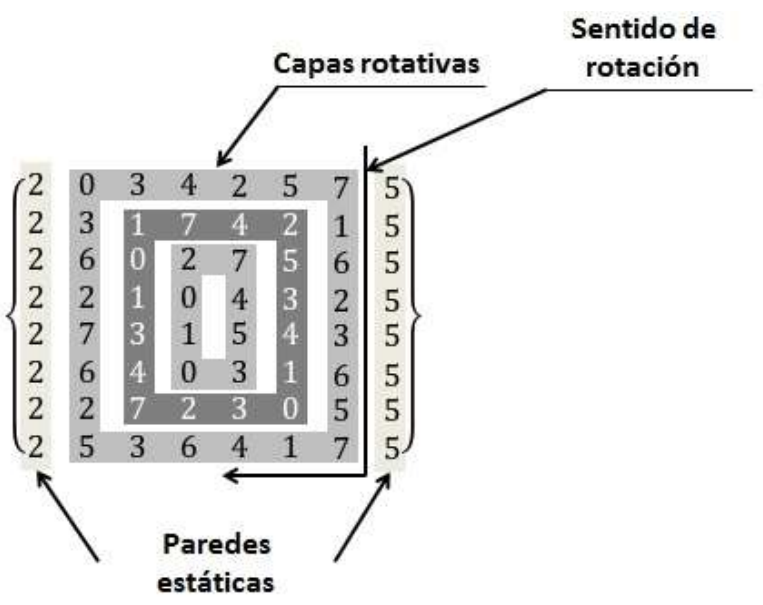

Fig. 3: Matriz poblacional en Simulated Annealing.

Existen muchos trabajos de aplicación del algoritmo en diferentes ámbitos, sin embargo en las telecomunicaciones y en especial en las redes ópticas los trabajos no han mejorado significativamente los resultados (Rodriguez et al., 2014; Rodriguez et al., 2015).

\section{Tabu Search (TS)}

El algoritmo TS fue presentado por primera vez por el Dr. Fred Glover en 1989, pero fue en 1990 cuando este algoritmo fue reconocido como una potente heurística. Se basa en el intercambio de acciones que mejoran o empeoran la FF, de tal forma que mediante un registro (memoria) de dichas acciones, se premian aquellas que la mejoran y se castigan las que empeoran la FF, de esta forma se busca converger rápidamente hacia el óptimo local (Ver figura 4). La principal ventaja del algoritmo es que cuando se localiza 
una posible solución esta converge rápidamente. Se basa en la idea que es mayor la información que brindan los errores que los aciertos, TS ha establecido récords en la búsqueda de mejores soluciones a los problemas de planificación de la producción y la programación, asignación de recursos, diseño de redes, enrutamiento, análisis financiero, las telecomunicaciones, la planificación de la cartera, gestión de la cadena de suministro, el modelado basado en agentes, el diseño de procesos de negocio, la previsión, el aprendizaje automático, minería de datos, etc. Muchos trabajos que utilizan este algoritmo hacen visible la robustez del método frente a alta carga de tráfico en escenarios dinámicos para redes ópticas WDM (Glover 1983; Glover 1989; Ye et al., 2014, Rodríguez et al., 2014).

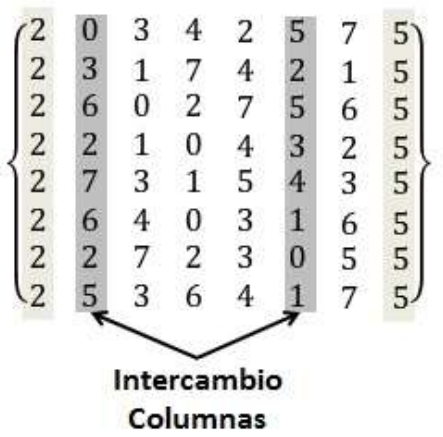

Fig. 4: Matriz poblacional en Tabu Search.

\section{Snake One (SNK1)}

El algoritmo Snake One es un algoritmo que utiliza la matriz de costo denominada matriz Snake (S), donde a través de desplazamientos horizontales y verticales, dicho algoritmo encuentra la ruta y asignación de longitud de onda con mucha eficiencia que el resto de heurísticas. Esto incluye saltos para evitar los ciclos cerrados, donde la máxima cantidad de saltos es inferior al número máximo en los nodos. Dicho algoritmo encuentra la ruta y asignación de longitud de onda con mucha eficiencia que el resto de heurísticas. La mayor desventaja del algoritmo es que por lo general utiliza nodos del perímetro de la red saturando con rapidez estos recursos, esta característica la coloca en desventaja ante escenarios de stress. En la figura 5, se muestra una red óptica de ejemplo con 8 nodos donde se indica que los enlaces inexistentes tienen un costo asociado muy grande (1000), de tal forma que las rutas que utilicen estos enlaces tendrán un costo total asociado grande y por lo tanto aparecerán al final de la matriz al ser ordenados descendentemente (Ver figura 5). De esta forma se garantiza una ruta de bajo costo pero no necesariamente la óptima.

\begin{tabular}{|c|c|c|c|c|c|c|c|c|c|}
\hline $\mathrm{N}$ & 0 & 1 & 2 & 3 & 4 & 5 & 6 & 7 & C \\
\hline 0 & 1000 & & (- & & 16 & 1000 & 1000 & 1000 & 5048 \\
\hline 1 & 16 & 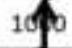 & 16 & ) & 1000 & 16 & 1000 & 1000 & 5048 \\
\hline & & & & & 00 & 000 & 000 & & 6032 \\
\hline 3 & 16 & 10 & 1000 & 100 & 1000 & 1000 & 16 & 0 & 6032 \\
\hline 4 & 16 & $10 \%$ & 1000 & 10 o & 1000 & 16 & 1000 & 0 & 6032 \\
\hline 5 & 1000 & 16 & 1000 & $1 d$ & 16 & 1000 & 1000 & 1фo & 6032 \\
\hline 0 & 1000 & 1000 & 1000 & & 100 & 500 & 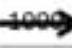 & & 6032 \\
\hline 7 & 1000 & 1000 & 16 & 1000 & 1000 & 1000 & 16 & 1000 & 6032 \\
\hline
\end{tabular}

Fig. 5: Algoritmo Snake One.

Los resultados mostrados en (Rodríguez et al., 2015) indican que mejora los indicadores de probabilidad de bloqueo pero a una elevada utilización de la red. Su comportamiento ante el stress (alto tráfico) es bastante mejor que el resto de heurísticas.

\section{Snake Two (SNK2)}

El algoritmo Snake Two, es una estrategia que utiliza el algoritmo Snake One para lograr el uso de los enlaces en su punto saturación, de esta forma dirigir el tráfico hacia ciertas zonas, dejando con cierta libertad otras zonas, de esta forma poder atender la demanda entrante. Esto se logra aplicando el algoritmo Snake One desde el origen de la solicitud hasta el enlace seleccionado y desde el enlace hacia el destino de la solicitud. El enlace seleccionado como más congestionado es obtenido de una matriz ordenada que monitoriza la carga de los enlaces y que periódicamente es ordenada ascendentemente, con la finalidad de tener siempre disponibles los más congestionados (Ver figura 6). 
Matriz de Enlaces Congestionados- MEC

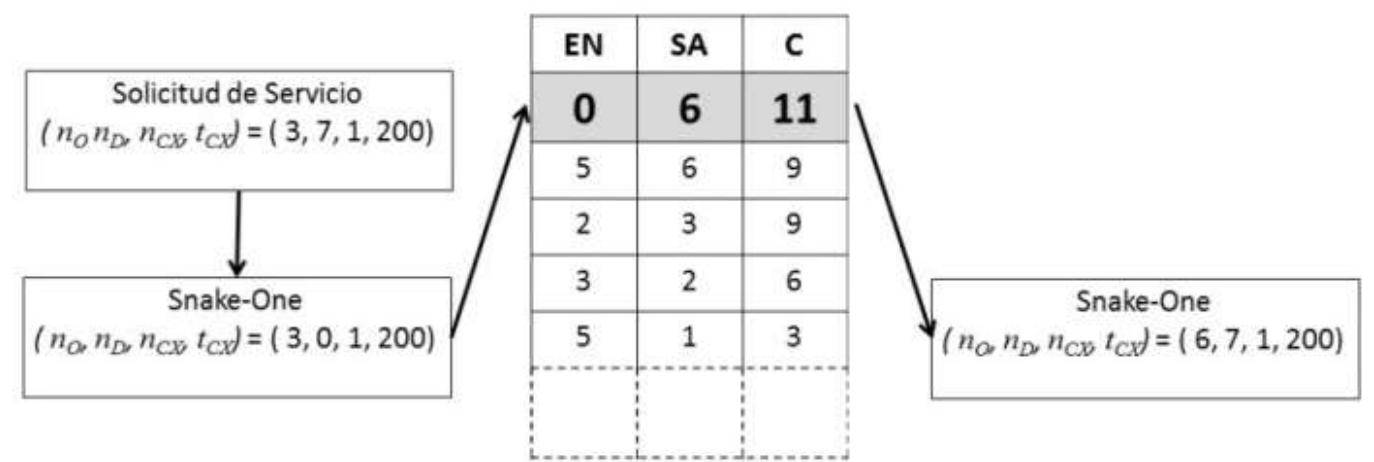

Fig. 6: Matriz de enlaces congestionados (MEC).

En la Figura 7, se observa la llegada de la solicitud R al OXC 3 y estando la MEC con el enlace 4-5 disponible se procede a calcular con Snake One la ruta del origen 3 al nodo 4 del enlace congestionado, obteniéndose 3-0-4; luego se procede a calcular la ruta del nodo 5 del enlace congestionado al destino 7 , obteniéndose 5-1-2-7; de esta forma se compone la ruta 3-0-4-5-1-2-7.

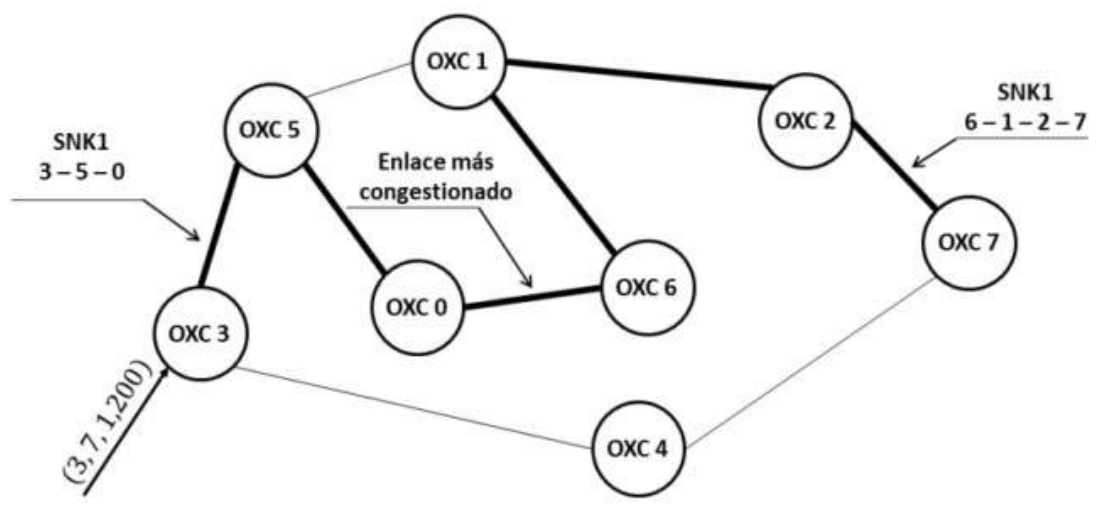

Fig. 7: Algoritmo Snake Two.

Los resultados mostrados indican que mejoran los indicadores promedio de probabilidad de bloqueo y utilización de la red (Rodriguez et al., 2015; Rodriguez et al., 2018).

\section{Otras Heurísticas}

El algoritmo PSO (Particle Swarm Optimization) fue introducido en 1995 por Reynolds y Heppner para modelar el comportamiento social, por ejemplo el movimiento de los peces las aves, logrando realizar observaciones donde el movimiento de uno o varios predomina sobre el resto (Kennedy et al., 1998; Markovic, 2016). El algoritmo busca la posición solución a través de la variación de las posiciones de las partículas corregidas en dirección por su velocidad, en cada iteración las partículas corrigen su posición con su mejor posición encontrada y con la mejor posición evaluada en el enjambre, de esta forma el movimiento total es la composición de todos las partículas pero con la dirección del líder similarmente cómo se comportan los enjambres. Se han realizado múltiples aplicaciones de este algoritmo en áreas como: Antenas, Biomédica, Redes de comunicaciones, Clustering y clasificación, Optimización Combinacional, Control, Diseño, Fallas, Finanzas, robótica y muchas más. Los resultados mostrados en estos artículos muestran la enorme utilización de este algoritmo cuya ventaja es la rápida convergencia en la solución pero a costos de un alto uso de los recursos y su desventaja es la improbabilidad de encontrar la solución óptima siempre (Poli, 2007; Poli 2008).

El algoritmo Firefly o Luciérnaga es introducido por Xin-She Yang en el 2008, basado en los procesos de atracción de las luciérnagas debido a su brillantez. Las condiciones del algoritmo son tres, la primera indica que todas las luciérnagas se atraen es decir no hay diferencia de género, la intensidad de la atracción es proporcional a la brillantez, si existe ausencia de brillantez las luciérnagas se mueven aleatoriamente. Este algoritmo es relativamente joven y en ciertas condiciones es similar al algoritmo PSO, su aplicación es numerosa y en los mismos ámbitos que los mencionados para PSO (Yang, 2010).

El algoritmo murciélago es introducido por Xin-She Yang (2010) y está basado en la ecolocación de micro murciélagos (microbats). El comportamiento con índices de pulso variable de emisión y amplitud. En 
términos prácticos, los murciélagos, sales en busca de su presa y según su ecolocación emite ondas de diferentes frecuencia y amplitud, siendo estas más intensas a medida que se acercan a su objetivo, de esta forma el grupo se comunica logrando obtener una o más presas (soluciones) en un solo proceso. Siendo aún muy joven se han realizado diferentes implementaciones con la finalidad de mejorar su desempeño (Vanegas et al., 2013; Yang, 2010; Yang, 2011; Yang et al., 2012).

\section{Algoritmo Snake Three (SNK3)}

El algoritmo Snake Three, es una nueva composición del algoritmo Snake One con un cambio en la estrategia; en este caso se utiliza el nodo más congestionado con disponibilidad para atender la solicitud, de esta forma se pretende dirigir el tráfico similarmente a Snake Two, pero esta vez hacia zonas de alto tráfico para lograr la máxima utilización de los recursos, esto es logrado a través del indicador de congestión nodal (ICN). La ecuación 9, muestra el indicador de congestión nodal del nodo $i$ que es la suma de los costos de los enlaces adyacentes.

$I_{C N}=\sum_{j=0}^{n} e_{i j} \quad \forall e_{i j} \exists$

En la Figura 8, se muestra el principio de funcionamiento de SNK3 a través del nodo más congestionado que es seleccionado de una matriz ordenada, que monitoriza la carga de los nodos y que periódicamente es ordenada ascendentemente, con la finalidad de tener siempre disponibles los nodos con mayor carga (Ver figura 8), para la selección del nodo se utiliza aquel de mayo costo que satisfaga la solicitud de conexión.

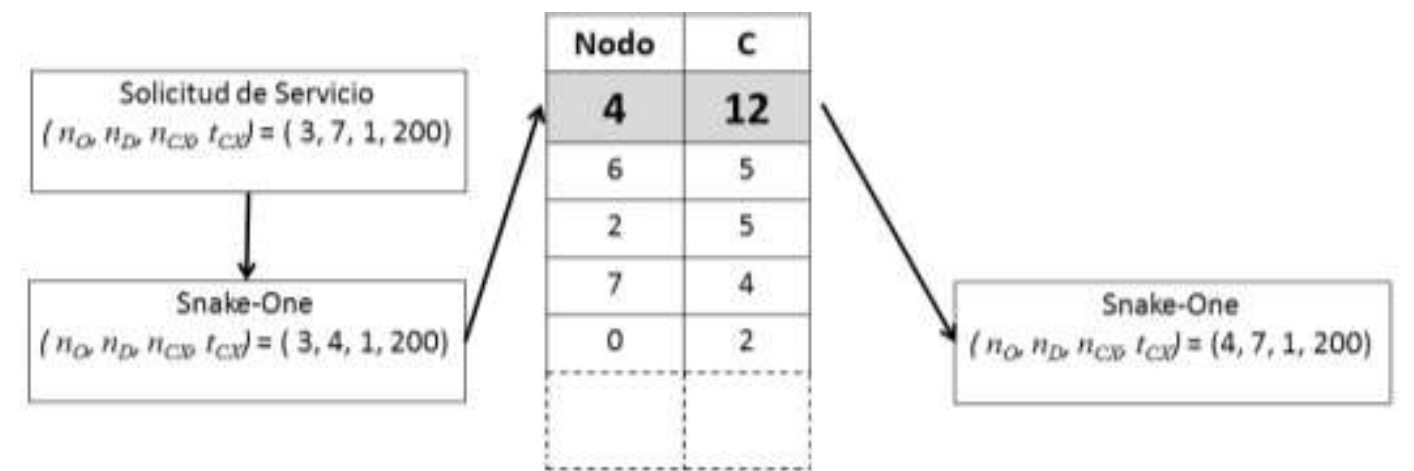

Fig. 8: Matriz de Nodos Congestionados (MNC).

En la Figura 9, se observa la ruta obtenida utilizando el algoritmo Snake One para calcular la ruta 3-0-1 hacia el nodo congestionado seleccionado y del nodo congestionado al destino de la solicitud calculando la ruta 1-2-7, de esta forma se compone la ruta 3-0-1-2-7. A diferencia de Snake Two, este algoritmo desarrolla rutas más cortas pero con el mismo efecto que Snake Two, lo que implica una menor utilización de la red.

En la Figura 10, se puede observar el diagrama de flujo de la metaheurística Snake Three donde se visibiliza el uso de Snake One (SO) y la Matriz de Nodos congestionados (MNC) por cada lambda disponible. Por otro lado, las matrices $\mathrm{C}, \mathrm{T}$, y $\lambda$ son actualizadas con la finalidad de mantener actualizada la MNC. Solo cuando se obtenga la ruta SO y coincida con la longitud de onda disponible se procederá a establecer el Lightpath (LP) en caso contrario se bloqueara la solicitud.

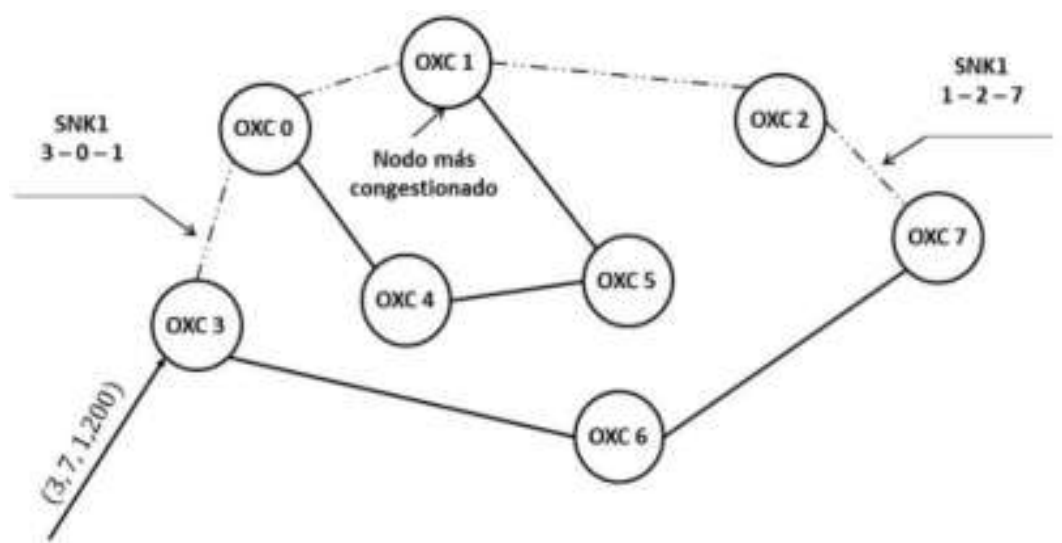

Fig. 9: Algoritmo Snake Three. 


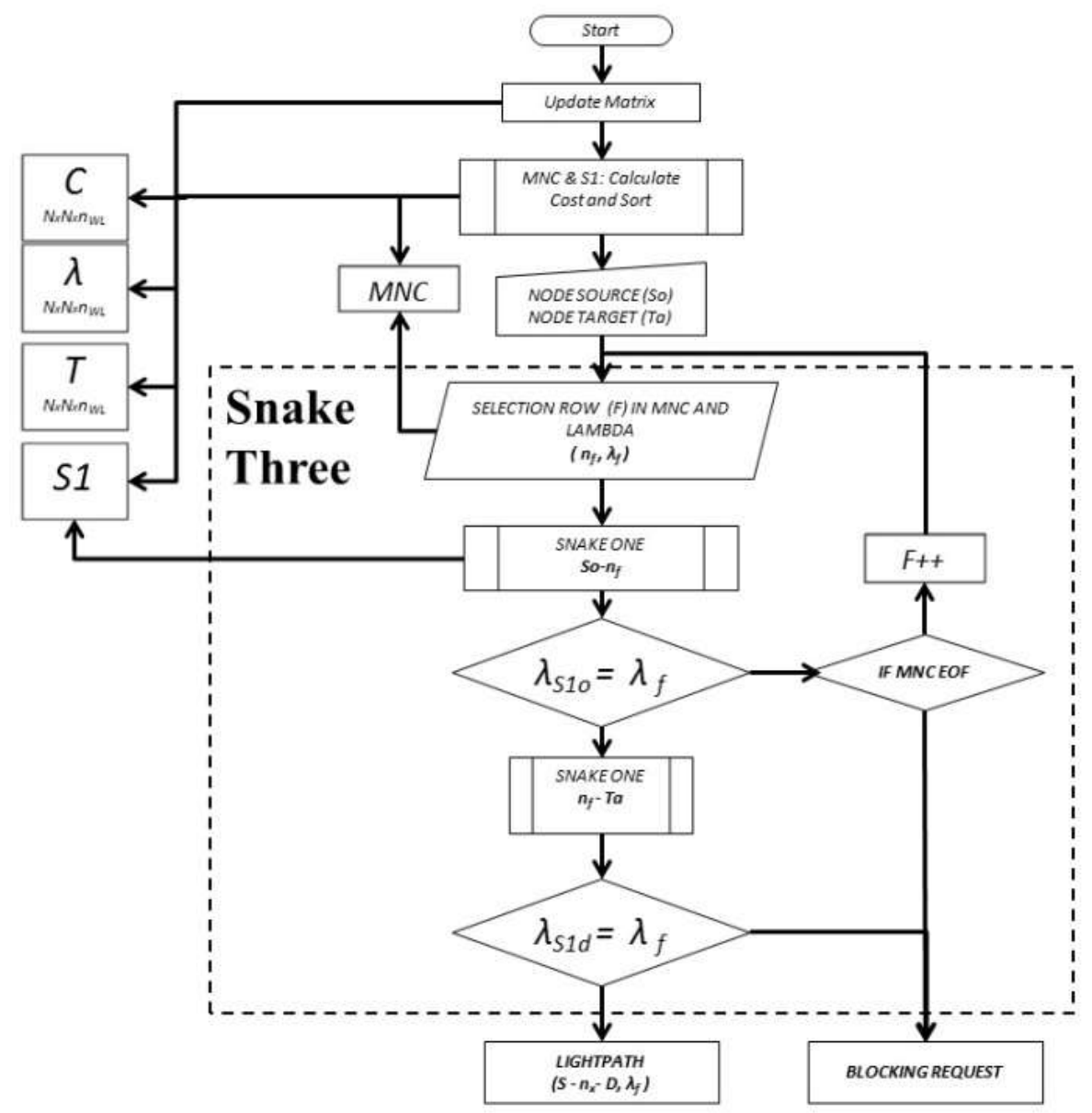

Fig. 10: Diagrama de flujo de Snake Three.

\section{Escenario de Simulación y demanda de tráfico}

La red óptica más utilizada para desarrollar simulaciones de redes WDM es la NSFNET o National Science Foundation Network (Ver figura 11), esta red se simulo con 14 nodos y 21 enlaces similarmente a los trabajos mostrados en (Rodriguez et al., 2014; Rodriguez et al., 2015) con la finalidad de realizar la comparación de los algoritmos heurísticos, a través de dos indicadores, Probabilidad de Bloqueo y Utilización de la Red, variando la carga de tráfico de 0 a 180 Erlangs. En la ecuación 10, se define la solicitud con tres parámetros que determinarán el tráfico. Donde $S_{m}^{i}$ determina la m-ésima solicitud de servicio del i-ésimo nodo, $\mathrm{n}_{d}$ determina el nodo destino solicitado, $\mathrm{n}_{c}$ determina el número de conexiones solicitadas y $t_{c}$ determina el tiempo de conexión solicitada.

$S_{m}^{i}=\left(\mathrm{n}_{d}, \mathrm{n}_{c}, t_{c}\right)$

Siendo $\mathrm{N}$ el número de nodos de la red $(\mathrm{N}=14)$ el número de total de solicitudes de simulación es de $10^{8}$ solicitudes. Los parámetros se eligen aleatoriamente según una distribución uniforme. El tiempo entre llegadas de solicitudes $t_{s}$ es de distribución exponencial de tal forma que el número de solicitudes que llegan al nodo es de distribución Possion. Los valores medios de estas variables aleatorias son similares a los valores utilizados en los trabajos (Rodriguez et al., 2014; Rodriguez et al., 2015) con fines de comparación, tales como número de longitudes de onda $n_{w}=8$, el tráfico es unifórmenle distribuido, el tiempo medio de solicitud de conexión es de $100 \mathrm{~ms}$.

Para establecer el tráfico dinámico se utiliza el índice de tráfico $I_{T}$ según la ecuación 11, si es menor que 1 la demanda es estática de lo contrario es dinámica, el presente trabajo desarrolla la simulación para el escenario dinámico y desarrolla una demanda que estresa la red de 0 a 180 Erlangs.

$\mathrm{I}_{T}=\frac{t_{S}}{t_{C}}$

El indicador Probabilidad de Bloqueo $\left(P_{b}\right)$ mide la probabilidad de una solicitud entrante de no ser atendida. Al existir la imposibilidad de espera de una solicitud esta es bloqueada al no ser atendida. Por otro lado el 
indicador Utilización de la Red $\left(U_{r}\right)$ mide la capacidad utilizada de la red a medida que se incrementa la carga en Erlangs. Esta carga es calculada multiplicando la tasa promedio de solicitudes entrantes con la tasa media de tiempo de conexión solicitado (100 ms).

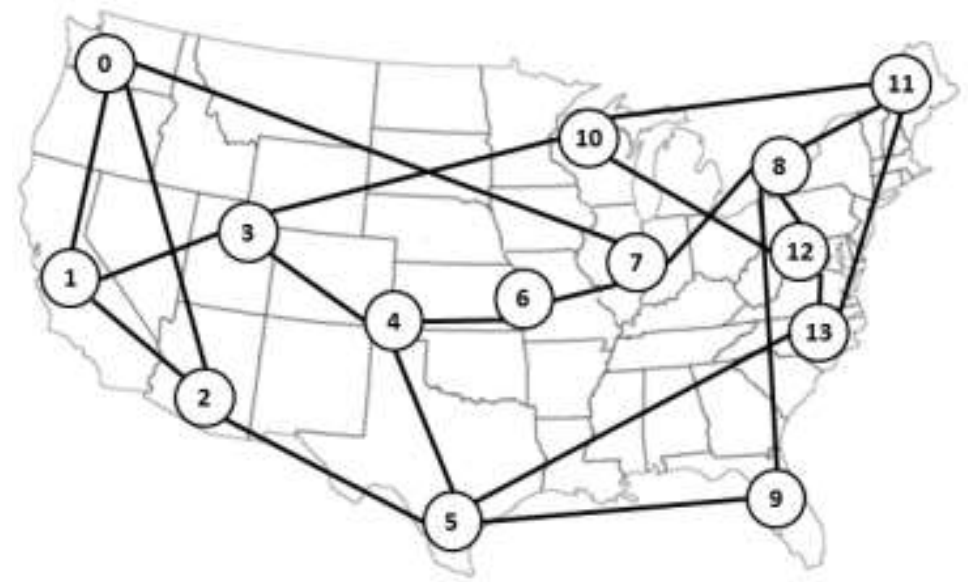

Fig. 11: Red NSFNET con 14 nodos y 21 enlaces.

\section{RESULTADOS}

Se utilizaron los indicadores Probabilidad de Bloqueo $\left(\mathrm{P}_{\mathrm{b}}\right)$ Utilización de la Red $\left(\mathrm{U}_{\mathrm{r}}\right)$ definidos anteriormente como elementos comparadores del desempeño algorítmico. En la Figura 12, se observa la probabilidad de bloqueo y el comportamiento de los algoritmos para diferentes cargas de simulación variando de 0 a 180 Erlangs. Se pueden distinguir tres intervalos de carga (Load Range of Blocking Probability - LRBP) que permiten explicar de mejor modo el comportamiento. El primer intervalo (LRBP1: 0-80 Erlangs) el segundo intervalo (LRBP2: 80-120 Erlangs) y el tercer intervalo (LRBP3: 120-180 Erlangs). LRBP1 representa un tráfico de baja carga, LRBP2 representa un tráfico transitorio y LRBP3 representa un alto tráfico o stress. Podemos observar que en LRBP1, SNK2 y SNK3 no ofrecen mayores diferencias comportándose similarmente superando al resto de heurísticas inclusive al algoritmo de referencia, mientras que en LRBP2 los comportamientos empiezan equilibrarse siendo TS, SNK1, SNK2 y SNK3 muy similares. Sin embargo, cuando el tráfico alcanza rangos de LRBP3 las heurísticas no responden mejor que el algoritmo de referencia, siendo este último el que mejor se desempeña. Es interesante resaltar el comportamiento de AS para altos niveles de tráfico siendo bastante parecido al algoritmo de referencia RF.

En la Figura 13, se observa la utilización de la red porcentualmente y se pueden distinguir dos intervalos de carga (Load Range of Network Utilization - LRNU) que permiten explicar de mejor modo el comportamiento. El primer intervalo (LRNU1: 0-40 Erlangs) el segundo intervalo (LRNU2: 40-180 Erlangs). Podemos observar que en LRNU1 los algoritmos heurísticos estudiados consumen más recursos que el algoritmo de referencia y en LRNU2 el uso de los recursos es creciente y constante, como se esperaba SNK3 encuentra rutas más cortas y consume menor recursos que la red que sus homólogos SNK1 y SNK2. Sin embargo no logra ser mejor que GA y SA, siendo el algoritmo de referencia el que menos recursos ocupa, cabe resaltar que el algoritmo de referencia no es heurístico y solo se utiliza con fines comparativos.

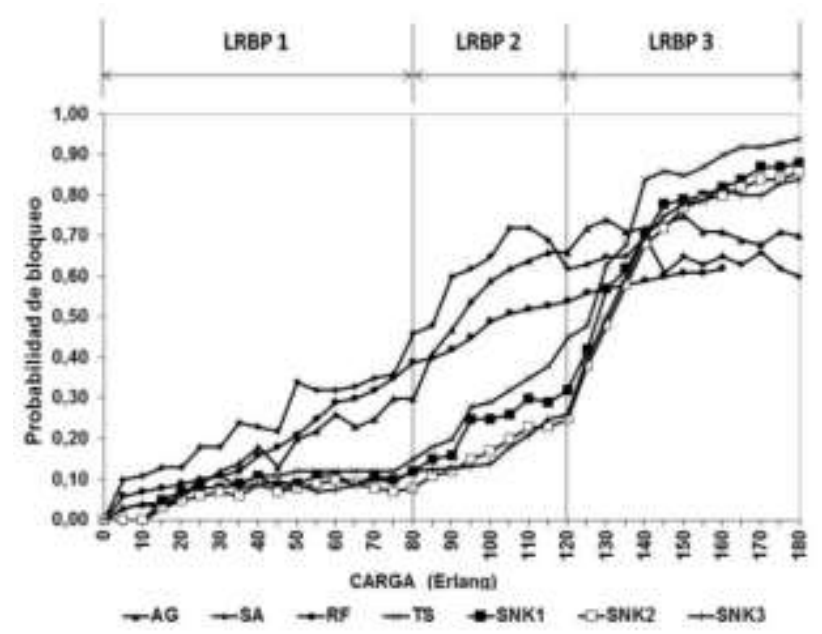

Fig. 12: Comparación de la Probabilidad de Bloqueo en la NSFNET. 


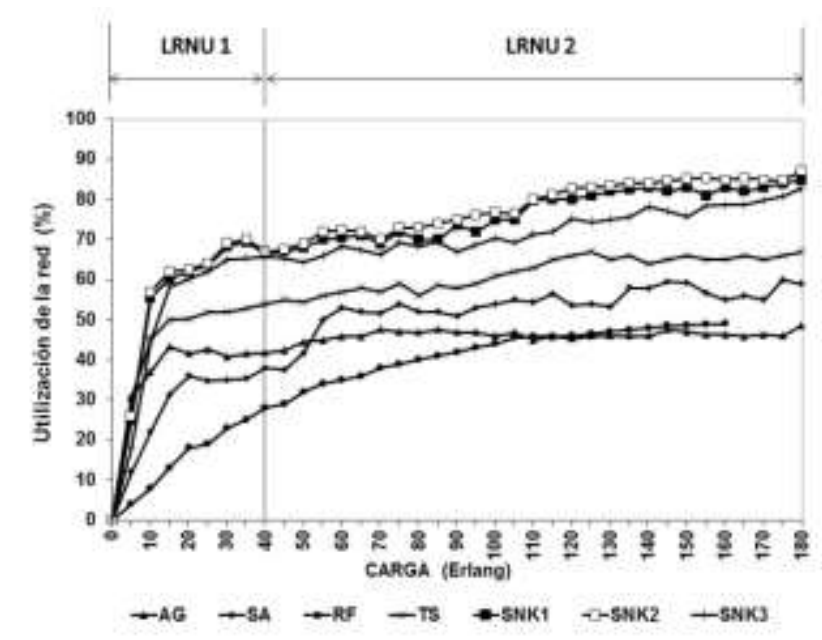

Fig. 13: Comparación de la utilización de red en la NSFNET.

\section{CONCLUSIONES}

El algoritmo de estudio se comparó con heurísticas que solo encuentran soluciones funcionales es decir, logran el objetivo de transportar sin ser necesariamente el óptimo y se utilizó como referencia un algoritmo determinístico (RF). Desde esta perspectiva, los trabajos mostrados y la metaheurística SNK3 tienen ventaja competitiva cuando observamos la probabilidad de bloqueo, instalándose como mejores respecto del algoritmo de referencia. Sin embargo, cuando observamos el indicador utilización de la red, se observa lo contrario, es decir el algoritmo de referencia (determinístico) es más eficiente.

Por otro lado, una de las ventajas más importantes de las heurísticas y metaheurísticas es la obtención de varias soluciones en el mismo proceso algorítmico que entrega robustez a la solución, mientras que los algoritmos convencionales optimizadores como el de referencia, solo encuentran una solución en el proceso, esto determina que frente a un sistema que requiera restauración los algoritmos metaheurísticas tendrán mayor oferta de solución. Esto permite concluir que para bajo rangos de tráfico (LRBP1 y LRNU1) las heurísticas responden con una baja probabilidad de bloqueo, pero a un alto consumo de los recursos de la red y este comportamiento empeora cuando el tráfico se eleva llegando a consumir mayores recursos que el algoritmo de referencia. Se deberá establecer nuevas simulaciones en un escenario de conversión de longitud de onda que permita disminuir las solicitudes bloqueadas $\sin$ un alto crecimiento de los recursos de la red.

\section{AGRADECIMIENTOS}

Especial agradecimiento para el Programa DYCYT de la Universidad de Santiago de Chile- USACH (Project DICYT No 061572RG), al Proyecto Basal USA1555 MECESUP-USACH y al Grupo de Investigación en Nuevas Tecnologías (GINT-DTI-USACH) por su importante apoyo al desarrollo de la investigación.

\section{REFERENCIAS}

Alarcón-Aquino, V.; Guerrero-Ojeda L.; Rodríguez-Asomoza J. y Rosas-Romero R., Análisis de Tráfico Autosimilar en Redes de Comunicaciones Usando Onditas (Wavelets), https://dx.doi.org/10.4067/S071807642005000200010, Inf. Tecnol., 16(2), 61-66 (2005)

Černý, V., Thermodynamical approach to the traveling salesman problem: An efficient simulation algorithm, doi:10.1007/BF00940812, Journal of Optimization Theory and Applications, 45(1), 41-51 (1985)

Chen, Y.; Hao, J. y Glover, F., A hybrid metaheuristic approach for the capacitated arc routing problem, doi: 10.1016/j.ejor.2016.02.015, European Journal of Operational Research, 253(1), 25-39 (2016)

Ferrucci, F. y Bock, S., Pro-active real-time routing in applications with multiple request patterns, doi:10.1016/j.eswa.2016.03.005, Expert Systems with Applications, 56(1), 320-334 (2016)

Galan-Jimenez, J. y Gazo-Cervero, A., Using bio-inspired algorithms for energy levels assessment in energy efficient wired communication networks, doi: 10.1016/j.jnca.2013.02.027, Journal of Network and Computer Applications, 37(1), 171-185(2014) 
Glover, F., Tabu Search — Part I, ORSA Journal on Computing, 1(3), 190-206 (1989)

Glover, F., Tabu Search — Part II, ORSA Journal on Computing, 2(1), 4-32 (1990)

Gutierrez, F.; Martin, E.; Perry, P.; Ellis, A.; Anandarajah, P. y Barry, L., WDM Orthogonal Subcarrier Multiplexing, doi: 10.1109/jlt.2015.2508418, Journal of Lightwave Technology, 34(8), 1815-1823 (2016)

Kennedy, J. y Eberhart, R., Particle Swarm Optimization, doi:10.1109/icnn.1995.488968, Proceedings of IEEE International Conference on Neural Networks, 4(1), 1942-1948 (1995)

Kirkpatrick, S.; Gelatt, C. y Vecchi, M.P., Optimization by Simulated Annealing, Science, doi:10.1126/science.220.4598.671, PMID 17813860, 220(4598), 671-680 (1983)

Lima, F.; Pereira, D.; Conceicao, S. y Nunez, N., A mixed load capacitated rural school bus routing problem with heterogeneous fleet: Algorithms for the Brazilian context, doi: 10.1016/j.ejor.2016.02.016, European Journal of Operational Research, 253(2), 356-371 (2016)

Lefever, W.; Aghezzaf, E. y Hadj-Hamou, K., A convex optimization approach for solving the single-vehicle cyclic inventory routing problem, doi:10.1016/j.cor.2016.02.010, Computers \& Operations Research, 72(1), 97-106 (2016)

Markovic, G., Wavelength Converters Placement in Optical Networks Using Bee Colony Optimization, doi: 10.4316/aece.2016.01001, Advances in Electrical and Computer Engineering, 16(1), 3-10 (2016)

Paraskevopoulos, D.; Bektas, T.; Crainic, T. y Potts, C., A cycle-based evolutionary algorithm for the fixedcharge capacitated multi-commodity network design problem, doi: 10.1016/j.ejor.2015.12.051, European Journal of Operational Research, 253(2), 265-279 (2016)

Piedrahita, E.; Hernández, C.; Pedraza, L. y Salcedo, O., Evaluación de Protocolos de Señalización en Multiprotocolo Generalizado de Conmutación de Etiquetas (GMPLS) Capaces de Conmutar Paquetes y Longitudes de Ondas, Inf. Tecnol., 25(1), 55-66 (2014)

Poli, R., An analysis of publications on particle swarm optimization applications, In Department of Computer Science, University of Essex, U.K., Technical Report CSM-469 (2007)

Poli, R., Analysis of the publications on the applications of particle swarm optimization, doi:10.1155/2008/685175, Journal of Artificial Evolution and Applications, 1(1), 1-10 (2008)

Rodriguez, A.; Gutierrez, A.; Rivera, L. y Ramirez, L., Comparing Genetic Algorithms and Simulated Annealing for dynamic traffic routing. Advanced Computer and Communication Engineering Technology, 315(1), 3-14 (2014)

Rodriguez, A.; Ramirez, L.; Rivera, L. y Gutierrez, A., Routing wavelength assignement: A solution with tabu search in dynamic traffic, Ingeniare. Rev. Chil. Ing., 22(4), 495-503 (2014)

Rodriguez, A.; Ramirez L. y Travieso, J., New heuristic algorithm for dynamic traffic in WDM optical networks, Revista Ingeniería e Investigación, 35(3), 100-106 (2015)

Rodriguez, A.; Travieso, J. y Savedra, F., DLE - WDM: Metaheurística para el Enrutamiento y Asignación de Longitud de Onda en Tráfico Dinámico, Ingeniare, en prensa, 26(1), (2018)

Russo, N.; Noriega, S. y Duchowicz, R., Implementación de Sistema Óptico para Grabado de Redes de Bragg en Fibra Óptica, Inf. Tecnol., 22(2), 121-130 (2011)

Sadiq, M.; Zhang, H.; O'Callaghan, J.; Roycroft, B. y otros seis autores, 40 Gb/s WDM Transmission Over 1.15-km HC-PBGF Using an InP-Based Mach-Zehnder Modulator, doi: 10.1109/jt.2015.2508941, Journal of Lightwave Technology, 34(8), 1706-1711 (2016)

Vanegas, S.; Amaya, I. y Correa, R., Algoritmo del murciélago virtual en el desarrollo de la Integral de Duhamel para sistemas estructurales con un grado de libertad, Revista Ingeniería de Construcción, 28(3), 278-289 (2013) 
Yang, X., Nature-inspired meta-heuristic algorithms, $2^{\text {nd }}$ Ed., Luniver Press, Beckington (2010)

Yang, X., New Metaheuristic Bat-Inspired Algorithm, Nature Inspired cooperative Strategies for Optimization, $1(1), 65-74(2010)$

Yang, X., Bat Algorithm for Multiobjective Optimization, International Journal of Bio-Inspired Computation, 3(5), 267-274 (2011)

Yang, X. y Gandomi, A.H., Bat Algorithm: A Novel Approach for Global Engineering Optimization, Engineering Computations, 29(5), 464-483 (2012)

Ye, A.; Zhang, Z.; Zhou, X. y Miao, F., Tabu Assisted Local Search for the Minimum Load Coloring Problem, doi: 10.1166/jctn.2014.3664, J. of Computational and Theoretical Nanoscience, 11(12), 2476-2480 (2014)

Zahedi, Z.; Akbari, R.; Shokouhifar, M.; Safaei, F. y Jalali, A., Swarm intelligence based fuzzy routing protocol for clustered wireless sensor networks, doi:10.1016/j.eswa.2016.02.016, Expert Systems with Applications, 55(1), 313-328 (2016)

Zang, H. y Jue, J., Dynamic Lightpath Establishment in Wavelength-Routed WDM Networks, IEEE Communications Magazine, 39(1), 100-108 (2001)

Zheng, Z.; Zhang F.; Wang D. y otros cuatro autores, Fiber Nonlinearity Mitigation in 32-Gbaud 16QAM Nyquist-WDM Systems, doi: 10.1109/jtt.2016.2535408, J. of Lightwave Technology, 34(9), 2182-2187 (2016) 
